\title{
Hipotensión permisiva en trauma
}

\author{
Yamileth Estrada Berrio a,1,2,3 ${ }^{\text {; Alejandro Barco Sánchez }}{ }^{\text {b,1,2 }}$; Sthefanía Ballén Ramírez ${ }^{\mathrm{c}, 2}$; \\ Jeimmy Lorena Salazar Bartolo ${ }^{\mathrm{d}, 2}$; John Jairo Ocampo Rincón ${ }^{\mathrm{e}, 3}$
}

\begin{abstract}
a Tecnóloga en Atención Prehospitalaria, Profesora Tecnología en Atención prehospitalaria „Facultad Ciencias de la salud, Estudiante de enfermería, ORCID: https://orcid.org/0000-0003-2053-0335, yestrada@utp.edu.co ; b Médico. Profesor Tecnología en Atención prehospitalaria ,Facultad Ciencias de la salud, ORCID: https://orcid.org/0000-0002-9315-7810, a.barco@utp.edu.co ; ' Estudiante de Tecnología en Atención prehospitalaria, ORCID: https://orcid.org/00000003-2916-7608, s.ballen@utp.edu.co ; ' Estudiante de Tecnología en Atención prehospitalaria, ORCID: https://orcid.org/0000-0003-0337-2646, 1.salazar1@utp.

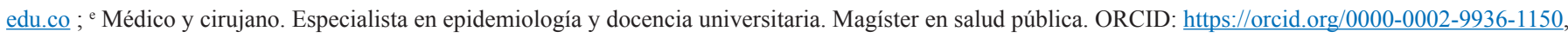
jiocampo@areandina.edu.co

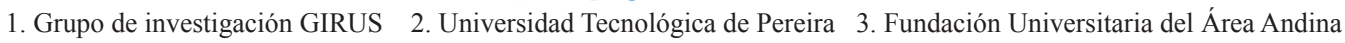
DOI https://doi.org/10.22517/25395203.24654

\section{Resumen}

\section{Introducción:}

El shock hemorrágico es una de las causas más comunes de muerte en pacientes con trauma debido a que pone en marcha un círculo vicioso de mecanismos que incluyen hipotermia, acidosis y coagulopatía. Para mitigar estos efectos, se han propuesto estrategias de control de daños, incluido el aporte controlado de líquidos con hipotensión permisiva contemplando metas en la presión arterial sistólica para mantener una adecuada perfusión de los tejidos.

Objetivo: Conocer información actualizada acerca del manejo de la hipotensión permisiva en pacientes con trauma.

Metodología: Se buscó información en las bases de datos Web-of-Science y Scopus de los últimos cinco años. El resultado arrojó un total de 118 artículos de los cuales se tomaron 30, según los criterios de inclusión y exclusión.

Resultados: Todos los artículos consideran el uso de la hipotensión permisiva como una buena opción para el manejo de los pacientes con trauma e hipotensión, sin embargo, difieren en qué momento es adecuado utilizarlo y en qué condiciones se debe realizar.

Conclusiones: Si bien la hipotensión permisiva se ha convertido en uno de los pilares fundamentales en el manejo prehospitalario del paciente con trauma, se requieren estudios investigativos en humanos para soportar cuándo y cómo debe utilizarse.

Palabras claves: Hipotensión permisiva, politrauma, control de daños.

\section{Permissive hypotension in trauma}

\begin{abstract}
Introduction: Hemorrhagic shock is one of the most common causes of death in trauma patients, because it sets in motion a vicious cycle of mechanisms, including hypothermia, acidosis, and coagulopathy. To mitigate these effects, damage control strategies have been proposed, including the controlled intake of fluids with permissive hypotension, contemplating goals in systolic blood pressure, and thus, maintaining adequate tissue perfusion.
\end{abstract}

Objective: The present research aimed to review the literature in search of updated information about the management of permissive hypotension in patients with trauma.

Methodology: Information was searched in the Web-ofScience and Scopus databases in the last five years. The result yielded a total of 118 articles, of which 30 were taken according to the inclusion and exclusion criteria.

Results: All the articles consider the use of permissive hypotension as a good option for the management of patients with trauma and hypotension, however, they differ when it is appropriate to use it and under what conditions it should be performed.

Conclusions: Although permissive hypotension has become one of the fundamental pillars in the prehospital management of the patient with trauma, research studies in humans are required to support when and how it should be used.

Keywords: Permissive hypotension, polytrauma, damage control. 


\section{Introducción}

A nivel mundial, el trauma genera alrededor de 5 millones de muertes al año (1,2 millones por accidentes de tránsito), con una carga global de enfermedad que hace que sea un importante problema de salud pública y uno de los motivos más frecuentes de consulta en los sistemas de salud latinoamericanos (1). Según el manual Advanced Trauma Life Support (ATLS) (2), la hemorragia es la causa más común de shock en pacientes con trauma. El shock hemorrágico pone en marcha un círculo vicioso de resultados, que incluye hipotermia, acidosis y coagulopatía; más conocidos como la tríada de la muerte. Identificar su origen, controlarlo y restaurar el volumen circulante son los objetivos principales en el tratamiento de este tipo de shock (2). Para lograr esto, se han propuesto estrategias como la reanimación de control de daños (RCD), que tiene como objetivo evitar las medidas terapéuticas desproporcionadas que puedan causar daños secundarios a los pacientes, optimizar la oxigenación de los tejidos y garantizar un flujo sanguíneo mínimo suficiente a los órganos vitales (evitando contrarrestar los mecanismos de coagulación); para esto, propone utilizar una reposición restringida de líquidos generando hipotensión permisiva, haciendo hincapié en la "reanimación equilibrada", con el objetivo de lograr una perfusión orgánica adecuada, evitar la sobrecarga circulatoria y atenuar el riesgo de resangrado (3). Sin embargo, los estudios han sido contradictorios o insuficientes en la determinación de esta metodología como la más adecuada, por lo que los desacuerdos se deben resolver bajo la evidencia actual antes de decidir el manejo sistemático del paciente (4). Aun así, el volumen de datos sobre reanimación hipotensiva continúa creciendo, por lo que se tratará de dilucidar en las siguientes páginas la eficacia, estrategias y datos estudiados a través de la literatura respecto a la reanimación hipotensiva en pacientes con shock hemorrágico traumático.

Durante los últimos años se ha investigado respecto al tratamiento más adecuado que se puede ofrecer a los pacientes con shock hemorrágico debido a trauma con el propósito de disminuir la mortalidad por este tipo de lesiones. Históricamente se ha reanimado a los pacientes con grandes cantidades de líquidos, pero se ha observado que, a pesar de los esfuerzos realizados, hay un riesgo de muerte considerablemente alto y las complicaciones han sido evidentes (5).

Por esto, actualmente varios estudios han buscado determinar el tratamiento adecuado para estos pacientes, introduciendo así el concepto de reanimación de control de daños que a su vez incluye la hipotensión permisiva, la cual consiste en el suministro de líquidos restringidos a un paciente con el fin de aumentar parcialmente la tensión arterial (sin alcanzar la normotensión) y así garantizar un flujo sanguíneo mínimo suficiente a los órganos vitales (6). Adicional a la hipotensión permisiva también se encuentra la reanimación hemostática (uso de sangre y productos sanguíneos) y la cirugía de control de daños (7). Se ha demostrado la efectividad de este método para disminuir la mortalidad en este tipo de trauma; sin embargo, no hay un consenso actual o una investigación con la suficiente validez estadística para apoyar el uso de esta metodología, a su vez que no se ha definido cuál sería la meta (en términos de presión arterial sistólica) de esta hipotensión permisiva (8). Muchos autores defienden la anterior estrategia basados en el hecho de que al corregir con grandes cantidades de líquidos la volemia perdida, se restaurará la función hemodinámica y permitirá una correcta perfusión de los tejidos y por tanto el paciente tendrá una mejor recuperación (9).

Aun así, los defensores de la reciente estrategia de reanimación de control de daños proponen que esta reanimación tan agresiva podría ir en contra de los mecanismos fisiológicos de coagulación, que buscan disminuir el sangrado y por tanto evitar mayor pérdida de volemia. El mecanismo propuesto es que este aumento de la presión (por la cantidad de líquidos suministrados) desplaza los coágulos que se están formando en los sitios de sangrado; empeora la acidosis, diluye los factores de coagulación, disminuye la viscosidad de la sangre y causa hipotermia en caso de que estos no fueran calentados previamente (10).

Pero en realidad, ¿Mejora la sobrevida en pacientes con trauma hemorrágico el uso de estrategias de reanimación basadas en hipotensión permisiva? En general los resultados arrojan evidencia de cierta mejoría en la supervivencia, sin embargo, debido a los estudios que no demuestran estos beneficios o que incluso concluyen que es contraproducente, se propone en el siguiente trabajo revisar en la literatura las investigaciones que hay hasta el momento y sacar la conclusión más adecuada.

Este artículo está basado en las investigaciones científicas que se han realizado sobre "hipotensión permisiva" en los últimos 5 años. Los datos obtenidos podrían permitir dilucidar cómo proporcionar una atención óptima del trauma hemorrágico en el ámbito prehospitalario, beneficiando tanto al personal de salud, mostrando las estrategias actualizadas para lograr el tratamiento más adecuado, así como a los pacientes. 


\section{Materiales y Métodos}

Se llevó a cabo una revisión sistemática de los estudios científicos publicados en revistas médicas, estos fueron identificados mediante búsquedas bibliográficas en las bases de datos Scopus y Web-of -Science para artículos publicados entre el 2015 y antes del 20 de abril de 2020. Se utilizaron los siguientes términos de búsqueda y el conector boleano AND: "Damage control" AND "Permissive hypotension" AND "Trauma". La búsqueda de literatura incluyó estudios originales integrativos (revisiones sistemáticas y meta-análisis), y artículos primarios (observacionales o ensayos clínicos). La intervención evaluada fue identificar cual es el manejo prehospitalario para los pacientes con trauma hemorrágico utilizando hipotensión permisiva y restricción de líquidos como tratamiento. La ecuación de búsqueda obtenida en las bases de datos se ilustra en la tabla 1.

\begin{tabular}{|c|c|}
\hline Base de datos & Ecuación de búsqueda \\
\hline Web of Science & $\begin{array}{l}\text { TEMA: ("Damage control" AND "Permissive hypotension" AND } \\
\text { "trauma") Refinado por: TIPOS DE DOCUMENTOS: (ARTICLE) } \\
\text { AND AÑOS DE PUBLICACIÓN: ( } 2020 \text { OR } 2019 \text { OR } 2018 \text { OR } 2017 \\
\text { OR 2016) Período de tiempo: Últimos } 5 \text { años. Índices: SCI- } \\
\text { EXPANDED, SSCI, A\&HCI, ESCI. }\end{array}$ \\
\hline Scopus & $\begin{array}{l}\text { TITLE-ABS-KEY ("Damage control" AND "Permissive } \\
\text { hypotension" AND "trauma") AND PUBYEAR > } 2015 \text { AND ( } \\
\text { LIMIT-TO (PUBYEAR, 2020) OR LIMIT-TO (PUBYEAR, } 2019 \\
\text { ) OR LIMIT-TO (PUBYEAR, 2018) OR LIMIT-TO (PUBYEAR } \\
\text {, 2017) OR LIMIT-TO (PUBYEAR, 2016)) AND (LIMIT-TO ( } \\
\text { LANGUAGE, "English")) }\end{array}$ \\
\hline
\end{tabular}

Tabla 1. Ecuación de búsqueda en bases de datos

Inicialmente se encontraron 118 artículos, identificados como estudios elegibles para su inclusión en el proceso de búsqueda (Fig. 1). Después de aplicar los filtros para la búsqueda (últimos 5 años y tipo de documento "artículo") quedaron 56 artículos, de los cuales 7 estaban duplicados y por lo tanto, se eliminaron de la lista, quedando 49 , posteriormente se excluyeron otros 19 artículos en vista de que su enfoque era diferente al respecto del manejo del trauma, e incluían aspectos relacionados con pacientes sangrantes en situaciones que no son de emergencia, artículos enfocados al área intrahospitalaria, así como artículos dirigidos a un entorno militar. Finalmente, se seleccionaron 30 artículos y se utilizaron en esta revisión sistemática.

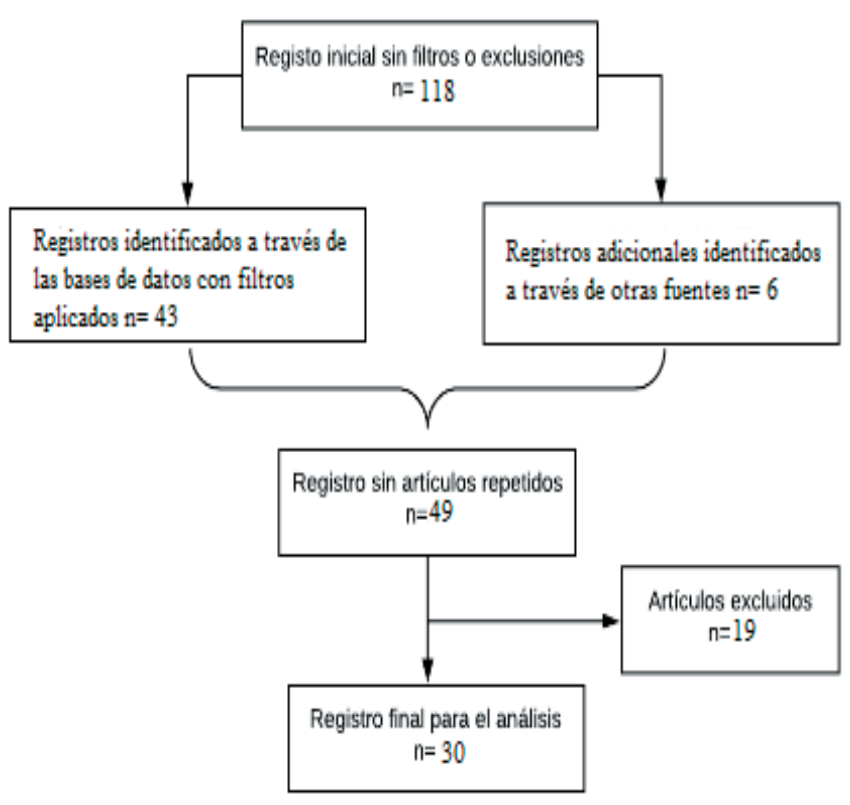

Figura 1. Esquema de análisis de artículos incluidos.

\section{Resultados}

Un 52\% de los artículos se basan en las publicaciones europeas que están enfocada en el manejo de trauma y utilizan como metas para la presión arterial sistólica valores entre $80-90 \mathrm{mmHg}$, argumentando que es la presión límite inferior aceptada para mantener una perfusión adecuada a los órganos (11), mientras que otros exponen que es mejor utilizar valores mayores a $100 \mathrm{mmHg}$ pero sin sobrepasar los $110 \mathrm{mmHg}$, ya que hay evidencia de que los valores por debajo de 90 y mayores a 110 al momento del ingreso aumentan el riesgo de mortalidad (12).

El 80\% de los artículos mencionan que está totalmente contraindicado el uso de este método de tratamiento en pacientes con lesión cerebral traumática, lesión medular y población anciana; debido a que una presión de perfusión cerebral adecuada es crucial para asegurar la oxigenación de los tejidos del sistema nervioso central lesionado y, con los parámetros de la hipotensión permisiva, estos requerimientos no se cumplen (13)(14).

En una revisión sistemática de la literatura realizada en el año 2018 con ensayos aleatorios controlados y estudios de cohorte, se encontró que la tasa de mortalidad entre los pacientes postraumáticos manejados con reanimación conservadora fue menor que la reanimación agresiva estándar, lo que 
indica que la reanimación con hipotensión permisiva puede crear una mejor tasa de supervivencia entre los pacientes traumatizados (15).

Dos artículos se enfocan en que esta estrategia no se debe utilizar mientras el foco de sangrado no esté controlado y solo hasta el momento en que se logre el control definitivo de este, teniendo en cuenta tiempos de traslado cortos (9) (16). Pues los tiempos tardíos en el traslado del paciente desde el lugar de los hechos hasta la llegada al hospital aumentan el riesgo de mortalidad (17). El deterioro del estado del paciente, es decir, el empeoramiento de una condición como la coagulopatía en un paciente que ha perdido una cantidad importante de volemia, está relacionado con un mal manejo de la emergencia, que incluye una administración inadecuada y excesiva de líquidos endovenosos sin llevar a cabo una reanimación que incluya todo el componente de control de daños (18).

Se ha mencionado el riesgo de que en medio de una reanimación agresiva con líquidos se acelere el proceso de coagulopatía, así como provocar complicaciones como la hipertensión intraabdominal secundaria oun síndrome compartimental abdominal, pues se encuentran estrechamente relacionado con la reanimación con líquidos de manera agresiva (19), sin embargo posterior a la reanimación con hipotensión permisiva o reanimación agresiva, se podrían presentar complicaciones como la lesión renal aguda, documentados en ambos casos (20) (21).

No hay un acuerdo sobre cuál es la mejor solución para reposición de líquidos, mientras unos apoyan la reanimación equilibradabajoelconceptode controlde daños, utilizando proporciones de plasma, plaquetas y glóbulos rojos que se aproximan a la sangre completa y volúmenes restringidos de cristaloides (22) (23), otros argumentan que el uso de productos sanguíneos prehospitalarios solo está disponible en algunos países y, por esto, los líquidos cristaloides siguen siendo la solución de elección en el medio prehospitalario (12). Se reconoce la necesidad de implementar la administración de cristaloides a bajo volumen en el escenario prehospitalario cuando el tiempo de traslado puede ser prolongado, de lo contrario, es preferible la rápida derivación a un centro hospitalario adecuado, donde se le brinde un tratamiento definitivo a las lesiones que generan la perdida de volemia (24). Respecto a las cantidades de líquidos que deberían ser administradas para lograr una aceptable presión arterial sistólica o generar un pulso radial de calidad, se ha recomendado un volumen de entre 250 a $500 \mathrm{~mL}$ (25). Los ensayos controlados aleatorios respecto al uso de una reanimación con líquidos agresiva en comparación con una reanimación hipotensiva en el medio prehospitalario e intrahospitalario, han respaldado los cambios en las guías internacionales del manejo de shock hemorrágico por trauma, sugiriendo unas presiones arteriales bajas, permitidas hasta que sea posible una reparación definitiva del daño, teniendo en cuenta características del paciente y de la lesión (26).

Actualmente no hay un consenso sobre las pautas a seguir en la reanimación para una atención prehospitalaria óptima, ya que sigue habiendo un debate considerable y continuo sobre cómo proporcionar la mejor atención al trauma en el ámbito prehospitalario. Es necesario el desarrollo de sistemas y protocolos de trauma, que puedan lograr mejoras en los resultados y aunque las estrategias de reanimación están en evolución, se requiere de mayor evidencia investigativa que las respalde (27) (28). Dentro de los resultados de investigaciones respecto al correcto manejo de líquidos en pacientes con shock hemorrágico por trauma se determina que una buena fluidoterapia es tan significativa como un manejo quirúrgico de la lesión (29), se hace importante énfasis en una buena valoración de los pacientes posterior a un trauma y así mismo una revisión a sus antecedentes patológicos que pudieran poner en riesgo al paciente con el uso de la hipotensión permisiva (30).

\section{Conclusiones}

La hipotensión permisiva se ha convertido en una de las principales estrategias de manejo del paciente en trauma demostrando su eficacia en la prevención de la coagulopatía dilucional y disminución del sangrado. Sin embargo, existe evidencia limitada para apoyar cuándo y cómo debe utilizarse. Gran parte de las practicas al respecto, se basan en evidencia observacional débil o en estudios en animales, siendo necesario pruebas sólidas de ensayos aleatorios controlados (ECA) en humanos. A medida que el conocimiento de la fisiopatología de la hemorragia masiva aumenta y la tecnología avanza, se espera que los protocolos de tratamiento racional continúen siendo refinados. Con este fin, se debe aspirar a realizar ensayos controlados aleatorios de alta calidad y coordinar los esfuerzos 
de investigación apuntando al objetivo de llevar más y mejor información al personal de salud prehospitalario e intrahospitalario, con lo cual se podrían tomar decisiones acertadas en el manejo del shock hemorrágico y la aplicabilidad de la hipotensión permisiva.

El personal intrahospitalario, principalmente en el servicio de urgencias, debe estar sintonizado con el manejo interdisciplinario del paciente con shock hemorrágico que requiere de un control de daños quirúrgico, para que desde su llegada hasta su disposición en quirófano se le haya manejado, no solo en el proceso de reparación de la lesión, sino también en su proceso postoperatorio, ya que la reanimación con control de daños mejora el proceso de recuperación.

\section{Referencias}

1. Byass P, de Courten M, Graham W, Laflamme L, McCaw-Binns A, Sankoh O, Zaba B. Reflections on the Global Burden of Disease 2010. PLOS Medicine. 2013; 10(7). doi:10.1371/journal. pmed.1001477

2. American College of Surgeons. Advance Trauma Life Support. 10th. ed. Chicago: American College of Surgeons; 2018.

3. Leidel BA, Kanz KG. Reanimation after trauma [Cardiopulmonary resuscitation in cardiac arrest following trauma]. Med Klin Intensivmed Notfmed. 2016; 111 (8): 695-702. doi: 10.1007/s00063-0160229-x.

4. Saracoglu A, Tetik S. Hemostatic Coagulation Management in Trauma. Int J Anestésico Anestesiol. 2018; 5 (2).doi.org/10.23937/2377-4630/1410080

5. Eick BG, Denke NJ. Resuscitative Strategies in the Trauma Patient: The Past, the Present, and the Future. J Trauma Nurs. 2018 Jul/Aug; 25(4):254263.doi: 10.1097/JTN.0000000000000383.

6. MacGoey P, Lamb CM, Navarro AP, Brooks AJ. Damage control: The modern paradigm. Trauma. 2016; 18 (3):165-177.doi: 10.1177 / 1460408616629507

7. Félix-Sifuentes DJ. Choque hipovolémico, un nuevo enfoque de manejo. Rev Mex Anest. 2018;
41(Suppl: 1):169-174.

8. Owattanapanich, N, Chittawatanarat, K, Benyakorn, T. et al. Risks and benefits of hypotensive resuscitation in patients with traumatic hemorrhagic shock: a meta-analysis. Scand J Trauma Resusc Emerg Med. 2018; 26 (107).

9. Palmer L. Fluid Management in Patients with Trauma: Restrictive Versus Liberal Approach. Vet Clin North Am Small Anim Pract. 2017 Mar; 47 (2):397-410. doi: 10.1016/j.cvsm.2016.10.014.

10. Schreiber MA, Meier EN, Tisherman SA, Kerby JD, Newgard CD, Brasel K, Egan D, Witham W, Williams C, Daya M, Beeson J, McCully BH, Wheeler S, Kannas D, May S, McKnight B, Hoyt DB; ROC Investigators. A controlled resuscitation strategy is feasible and safe in hypotensive trauma patients: results of a prospective randomized pilot trial. J Trauma Acute Care Surg. 2015 Apr; 78(4):687-95; discussion 695-7.doi: 10.1097/ TA.0000000000000600.

11. Spahn DR, Bouillon B, Cerny V, Duranteau J, Filipescu D, Hunt B, Rossaint, R. The European guideline on management of major bleeding and coagulopathy following trauma: fifth edition. Critical Care. 2019. 23 (98). https://doi.org/10.1186/s13054-019-23473.

12. Woolley T, Thompson P, Kirkman E, Reed R, Ausset S, Beckett A, Bjerkvig C, Cap AP, Coats T, Cohen M, Despasquale M, Dorlac W, Doughty H, Dutton R, Eastridge B, Glassberg E, Hudson A, Jenkins D, Keenan S, Martinaud C, Miles E, Moore E, Nordmann G, Prat N, Rappold J, Reade MC, Rees P, Rickard R, Schreiber M, Shackelford S, Skogran Eliassen H, Smith J, Smith M, Spinella P, Strandenes G, Ward K, Watts S, White N, Williams S. Trauma Hemostasis and Oxygenation Research Network position paper on the role of hypotensive resuscitation as part of remote damage control resuscitation. J Trauma Acute Care Surg. 2018 Jun; 84(6S Suppl 1):S3-S13. doi: 10.1097/ TA.0000000000001856.

13. Muckart DJJ. Damage Control Resuscitation: Restarting the Machinery of Life. Curr Trauma Rep. 2018, 4: 199-204. https://doi.org/10.1007/ s40719-018-0129-z.

14. Harada MY, Ko A, Barmparas G, Smith EJ, Patel BK, Dhillon NK, Thomsen GM, Ley EJ. 10-Year 
trend in crystalloid resuscitation: Reduced volume and lower mortality. Int J Surg. 2017 Feb; 38:78-82. doi: 10.1016/j.ijsu.2016.12.073.

15. Albreiki M, Voegeli D. Permissive hypotensive resuscitation in adult patients with traumatic haemorrhagic shock: a systematic review. Eur J Trauma Emerg Surg. 2018 Apr; 44(2):191-202. doi: 10.1007/s00068-017-0862-y.

16. Weigeldt M, Stengel D, Ekkernkamp A, Maegele M. Coagulation management in bleeding trauma patients. Trauma und berufskrankheit. 2018. 20 (3): 209-215. doi:10.1007/s10039-018-0390-y

17. Bores SA, Pajerowski W, Carr BG, Holena D, Meisel ZF, Mechem CC, Band RA. The Association of Prehospital Intravenous Fluids and Mortality in Patients with Penetrating Trauma. J Emerg Med. 2018 Apr; 54(4):487-499.e6. doi: 10.1016/j. jemermed.2017.12.046.

18. Simmons JW, Powell MF. Acute traumatic coagulopathy: pathophysiology and resuscitation. Br J Anaesth. 2016 Dec; 117(suppl 3):iii31-iii43. doi: $10.1093 / \mathrm{bja} / \mathrm{aew} 328$.

19. Duchesne JC, Kaplan LJ, Balogh ZJ, Malbrain ML. Role of permissive hypotension, hypertonic resuscitation and the global increased permeability syndrome in patients with severe hemorrhage: adjuncts to damage control resuscitation to prevent intra-abdominal hypertension. Anaesthesiol Intensive Ther. 2015; 47(2):143-55.doi: 10.5603/ AIT.a2014.0052.

20. Ho K.-H, Tarng YW, Lin HL. Permissive Hypotensive Resuscitation in Patients with Traumatic Hemorrhagic Shock. Scand J Trauma Resusc Emerg Med. 2019; 27 (14). https://doi.org/10.1186/ s13049-019-0595-5

21. Pohlman TH, Fecher AM, Arreola-Garcia C. Optimizing transfusion strategies in damage control resuscitation: current insights. J Blood Med. 2018 Aug 20; 9:117-133. doi: 10.2147/JBM.S165394.

22. Cantle PM, Cotton BA. Balanced Resuscitation in Trauma Management. Surg Clin North Am. 2017 Oct; 97(5):999-1014. doi: 10.1016/j. suc.2017.06.002.

23. Kalkwarf KJ, Cotton BA. Resuscitation for Hypovolemic Shock. Surg Clin North Am.
2017 Dec; 97(6):1307-1321. doi: 10.1016/j. suc.2017.07.011.

24. Ramesh GH, Uma JC, Farhath S. Fluid resuscitation in trauma: what are the best strategies and fluids?. Int J Emerg Med. 2019; 12 (38). https://doi. org/10.1186/s12245-019-0253-8

25. Gondek S, Schroeder ME, Sarani B. Assessment and Resuscitation in Trauma Management. Surg Clin North Am. 2017 Oct; 97(5):985-998. doi: 10.1016/j. suc.2017.06.001.

26. Carrick MM, Leonard J, Slone DS, Mains CW, Bar-Or D. Hypotensive Resuscitation among Trauma Patients. Biomed Res Int. 2016. doi: 10.1155/2016/8901938.

27. Giannoudi M, Harwood P. Damage control resuscitation: lessons learned. Eu J Trauma Emerg Surg. 2016; 42(3): 273-282. doi: 10.1007/s00068015-0628-3.

28. Dauer E, Goldberg A. What's New in Trauma Resuscitation? Adv Surg. 2019 Sep; 53:221-233. doi: 10.1016/j.yasu.2019.04.010.

29. Chang R, Holcomb JB. Optimal Fluid Therapy for Traumatic Hemorrhagic Shock. Crit Care Clin. 2017 Jan; 33(1):15-36. doi: 10.1016/j.ccc.2016.08.007.

30. Das JM, Anosike K, Waseem M. Permissive Hypotension. StatPearls. 2020. Availabre from: https://www.ncbi.nlm.nih.gov/books/NBK558915/ 